Cite this: RSC Adv., 2017, 7, 11304

Received 17th November 2016

DOI: $10.1039 / c 6 r a 26919 b$

rsc.li/rsc-advances

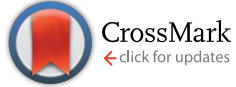

Accepted 2nd February 2017

\section{Elucidation of hierarchical metallophthalocyanine buffer layers in bulk heterojunction solar cells $\uparrow$}

\begin{abstract}
M. Alam Khan *a $^{* a}$ and Umme Farva ${ }^{b}$
Vapour deposited metallophthalocyanines (VoPC, NiPC, SnPC, TiPC, FePC, InPC) were inserted as thin ptype buffer layers into organic solar cells consisting of regio-regular poly(3-hexylthiophene) and $[6,6]$ phenyl C61-butyric acid methyl ester in order to modify the band alignment for enhanced photovoltaic responses. It is observed that the VoPC, NiPC and SnPC show higher efficiencies in both $\sim 5 \mathrm{~nm}$ and $\sim 10 \mathrm{~nm}$ thicknesses of film when coated on a PEDOT:PSS layer. The observations were an enhanced efficiency with a maximum $J_{S C}$ value of $14.80 \mathrm{~mA} \mathrm{~cm}^{-2}, V_{O C}$ of 0.60 , and $\mathrm{FF}$ of $51 \%$ with a conversion efficiency of $3.61 \%$ in the case of $\sim 5 \mathrm{~nm}$ thick VoPC, while the $\sim 5 \mathrm{~nm}$ thick NiPC shows a $J_{\mathrm{SC}}$ value of $11.15 \mathrm{~mA} \mathrm{~cm}{ }^{-2}$ with $3.13 \%$ efficiency, and similarly the $\sim 10 \mathrm{~nm}$ thick NiPC has a $J_{\mathrm{SC}}$ value of $8.98 \mathrm{~mA}$ $\mathrm{cm}^{-2}$ with overall efficiency of $2.52 \%$. However, the TiPC, FePC and InPC show poor efficiencies even lower than those of the usual bulk-heterojunction solar cells with deposition of either a $\sim 5 \mathrm{~nm}$ or a $10 \mathrm{~nm}$ buffer layer. The enhanced efficiency in VoPC, NiPC and SnPC may be the result of extended absorption, high transparency, planar porphyrin complex orientation and holding $\mathrm{p}$-type properties to transfer the hole efficiently towards the anodes, with additionally a better bicontinuous interpenetrating network with vapour deposited layers, which greatly reduces the exciton loss and improves the charge transport capability.
\end{abstract}

\section{Introduction}

Photovoltaic (PVs) devices are perceived as a sustainable and viable means of meeting the scorching needs of the future global energy appetite with a minimal detrimental effect on the environment. Organic photovoltaics (OPV) are a promising and attractive concept, consisting of a light-harvesting regio-regular conjugated polymer and electron accepting fullerenes ${ }^{\mathbf{1}}$ as an alternative to silicon, CIS, perovskite and CZTS solar cells, owing to their light weight, solution processability, large area, cost-effectiveness and flexibility. ${ }^{2,3}$ However, it is understood and validated that the efficiency behaviour of bulkheterojunction (BHJ) solar cells is very sensitive to small changes in material blend ratios, ${ }^{4}$ percentage variations in the composition, the solvent used for spin casting, ${ }^{\mathbf{5}, \mathbf{6}}$ and changes in post fabrication treatments such as the time and/or temperature of annealing, ${ }^{7}$ which can dramatically influence the performance of the final device. Moreover, it has also been

${ }^{a}$ Research Center for Solar Energy Chemistry, Osaka University, 1-3 Machikaneyama, Toyonaka Campus, Osaka 560-8531, Japan.E-mail: alamkhan77@gmail.com

${ }^{b}$ Research Center for Ultra Precision Science and Technology, Osaka University, 2-1 Yamadaoka, Suita, Osaka 565-0871, Japan

$\dagger$ Electronic supplementary information (ESI) available. See DOI: 10.1039/c6ra26919b

\$ Present affiliation: Department of Energy and Materials Engineering, Dongguk University-Seoul, Seoul 100-715, Republic of Korea, Ph: +82-2-2123-5842, Fax: +82-2-2123-5375. reported that the donor and acceptor phases should be bicontinuous with a characteristic exciton diffusion length of $\sim 10 \mathrm{~nm}$ in order to efficiently hop the dissociated excitons towards the respective electrodes, resulting in appreciable photocurrents. ${ }^{\mathbf{8} 9}$ So, to date it remains a challenge to maximize photon absorption, charge separation and charge transport while maintaining the necessary size scale and continuity constraints. The charge carrier photogeneration in organic solar cells starts with intramolecular excitation leading to a localized Frenkel exciton ${ }^{\mathbf{1 0}}$ where an electron acceptor with a high electronic affinity and an electron donor with a high ionization energy are separated in the time scale of femto seconds $(\sim 50 \mathrm{fs})^{\mathbf{1 1}}$ which is much faster than other competing processes like photoluminescence (ns) and charge recombination ( $\mu \mathrm{s})$. However, the formed geminate polaron pairs, which were not separated at the interface between the electron donor and the electron acceptor owing to the intrinsic characteristic of polymer molecules, have a high trap density, resulting in a dead end in the transport net and soon the mobility is low and unbalanced. ${ }^{12}$ One interesting way to improve the overall $\mathrm{BHJ}$ solar cell efficiency is to enhance the $J_{\mathrm{SC}}$ value by extending the light absorbance in the standard solar spectrum (AM 1.5G).

Enhancing the hole mobility in the P3HT phase of the bulkheterojunction solar cell might be an interesting factor resulting in an improved efficiency; for instance, the hole mobility can be dramatically increased by more than three fold up to 4.4 $\times 10^{-2} \mathrm{~cm}^{2} \mathrm{~V}^{-1} \mathrm{~S}^{-1}$ by employing several strategies. ${ }^{13-15}$ It has 
been also documented that introducing an optical spacer into the device is another approach to trap the incident photons, but the optical spacer does not always bring an observable improvement in efficiency as a result of weak electrical transport, poor carrier collection or sub-band-gap transmission and the thermalization of the hot charge carriers. ${ }^{16}$ In the beginning and to date PEDOT:PSS is an important and frequently used buffer layer focused on the improvement in hole mobility at the anode side, since the hole mobility of P3HT $\left(5.0 \times 10^{-11} \mathrm{~m}^{2} \mathrm{~V}^{-1} \mathrm{~S}^{-1}\right)$ is 4000 times slower than the electron mobility of PCBM $\left(2.0 \times 10^{-7} \mathrm{~m}^{2} \mathrm{~V}^{-1} \mathrm{~S}^{-1}\right)$ in an organic solar cell which might be one of the glitches that can be rectified for an improvement in solar cell efficiency. It is found that the conductivity, morphology and work function of PEDOT:PSS are influenced by post-deposition treatment ${ }^{17}$ and, thus, high temperature treatment can also change the paracrystalline structure.$^{18}$ Furthermore, the doping of PEDOT:PSS with different components, such as sorbitol, glycerol, ${ }^{\mathbf{1 7}}$ and mannitol, ${ }^{19}$ could control the conductivity, resulting in enhanced solar cell efficiency. The conformation of PEDOT chains in the films changed when ethylene glycol, mesoerythritol and 2-nitroenthanol were doped, resulting in an increase in PEDOT:PSS conductivity. ${ }^{20}$ On doping glycerol into PEDOT:PSS, up to a hundred times enhancement in conductivity and a lower work function were observed as compared to the un-doped films. ${ }^{17}$

The metallophthalocyanines are a group of structural porphyrin complexes which have been mostly used in the areas of spintronic, electrochromic, non-linear optical devices, information storage systems and liquid crystal applications. ${ }^{21-25}$ Their thermally stable nature and unique optoelectronic properties make them an appropriate choice for thin film deposition by vapour phase techniques. Hipps and co-workers demonstrated that the d-orbital occupation of metallophthalocyanines had a dramatic effect on the central metal ion $^{26,27}$ and appears as either a hill or a dip, relative to the surrounding phthalocyanine ring, depending upon the occupation of the $\mathrm{d}_{z}{ }^{2}$ orbital. Tao studied electron transfer through redox-active molecules adsorbed on a conductive substrate with a scanning tunneling microscope (STM) in aqueous solution ${ }^{28}$ and found that by adjusting the substrate potential, the Fermi levels of the substrate and tip were shifted relative to the energy levels of the molecules. Aligning the Fermi levels to a molecular energy level produced a 10 -fold increase in the tunneling current that flows between the substrate and the scanning tunneling microscope (STM) tip in the atomic surface analysis. Inspired by these ideas of an enhanced current in our effort towards interface engineering we have applied several transition metallophthalocyanines (VoPC, NiPC, CuPC, SnPC, TiPC, FePC, InPC) in order to elucidate and improve the power conversion efficiencies in bulk-heterojunction solar cells. Since it has been reported that the separation of the HOMO and LUMO orbitals of most phthalocyanine rings is about $1.5-2.4 \mathrm{eV},{ }^{29}$ such chargetransfer transitions may lie at very low energies absorbed in the visible region, and hence may also extend the solar cell absorption which may improve the overall efficiency and ameliorate charge transfers to anodes, owing to their weak ptype nature.

\section{Experimental}

\subsection{Method and preparation}

The bulk-heterojunction (BHJ) organic solar cells in this study were fabricated by using reagent grade chemicals such as poly(3-hexylthiophene-2,5-dilyl) (P3HT) and [6,6]-phenyl $\mathrm{C}_{61}$ butyric acid methyl ester (PCBM) purchased from the Aldrich company. First, the ITO (resistivity $15 \mathrm{~V} \mathrm{sq.}^{-1}$ ) glass was masked, etched and cleaned with detergent and ethanol, then ultrasonicated thrice in acetone and afterwards dried with blown $\mathrm{N}_{2}$ at $80{ }^{\circ} \mathrm{C}$ for $2 \mathrm{~h}$, and cleaned further with $\mathrm{O}_{2}$ plasma $\left(40 \mathrm{~W}, 7.5 \times 10^{-5} \mathrm{~Pa}\right)$ for $15 \mathrm{~min}$. Then the highly conducting poly(3,4-ethylenedioxylenethiophene):polystyrene sulphonic acid (PEDOT:PSS), which was purchased from Baytron (H. C. Stark), was spin casted for $3000 \mathrm{rpm}$ for $40 \mathrm{~s}$ on the etched ITO glass after passing through a $0.80 \mu \mathrm{m}$ filter. After that the substrate was baked at $120{ }^{\circ} \mathrm{C}$ for 20 minutes under a vacuum. Then the PEDOT:PSS coated glass was placed in the chamber for the different applications of a p-type buffer layer of metallophthalocyanines by thermal evaporation at a pressure of $\sim 5 \times$ $10^{-5}$ Torr and evaporated with $\sim 5 \mathrm{~nm}$ or $\sim 10 \mathrm{~nm}$ thin layers (in the case of copper phthalocyanines they were $3 \mathrm{~nm}, 5 \mathrm{~nm}, 7 \mathrm{~nm}$, $10 \mathrm{~nm}$ and $20 \mathrm{~nm}$ ) in each case. Chloroform was used as solvent for the active layer application. Then $1.0 \mathrm{ml}$ of solvent P3HT (10 $\mathrm{mg}$ ) and PCBM (10 mg) were added into a $4 \mathrm{ml}$ vial in a glove box and sonicated for $15 \mathrm{~min}$ to dissolve it until a homogeneous solution was visually observed. Then the solution was further magnetically stirred for $24 \mathrm{~h}$ (aging). After that the active layer was spin casted over the ITO/PEDOT:PSS/metal phthalocyanines (MOPC) layer at $1000 \mathrm{rpm}$ for $30 \mathrm{~s}$ and then dried at room temperature. After that using a cotton bud we removed the P3HT:PCBM spillover at the PN electrodes. To make the cell simpler without the utilization of thin $\mathrm{LiF}$, an $\mathrm{Al}(80 \mathrm{~nm})$ electrode was deposited by thermal evaporation in a vacuum of about $2 \times 10^{-3}$ Torr. Then post annealing was carried out in a vacuum at $75{ }^{\circ} \mathrm{C}$ for $15 \mathrm{~min}$. Devices with different layer compositions were fabricated as ITO/PEDOT:PSS/P3HT:PCBM/ $\mathrm{Al}$ denoted as normal BHJ, ITO/PEDOT:PSS/MOPC/ P3HT:PCBM/Al, and ITO/PEDOT:PSS/P3HT:PCBM/MOPC/Al.

\subsection{Characterization}

The characterization of the fabricated devices was done by ultraviolet visible (UV-vis) absorption spectra obtained by using a Hitachi U-4100 UV/vis/NIR spectrometer and scanning electron microscope (SEM) images by a TESCAN MIRA LMH2 microscope. The current-voltage $(J-V)$ of the solar cells was measured in air with a Bunkoh-Keiki CEP-015 photovoltaic measurement system under simulated AM 1.5G irradiation (100 $\mathrm{mW} \mathrm{cm} \mathrm{cm}^{-2}$ ). The light intensity was calibrated to $100 \mathrm{~mW} \mathrm{~cm}^{-2}$ using a calibrated standard silicon solar cell and KG5 filter. An array device, with each array having an active area of $0.02 \mathrm{~cm}^{2}$, was fabricated on the electrochemically grown substrates and tested under ambient conditions. 


\section{Results and discussion}

Fig. 1a shows the UV-visible spectra of the as-grown thin films by the vapour deposition of copper phthalocyanines (CuPC) as a representative $\mathrm{p}$-type buffer layer on the glass/ITO/PEDOT:PSS surface to elucidate the optimum thickness $(3 \mathrm{~nm}, 5 \mathrm{~nm}, 7 \mathrm{~nm}$, $10 \mathrm{~nm}$, or $20 \mathrm{~nm}$ ) in order to get the best solar cell performance. It has been documented that the application of such a CuPC thin buffer layer causes an enhanced efficiency in the tandem type of organic solar cell. ${ }^{30}$ It can be observed from the figure that the enhanced absorbance successively noted with respect to the thickness had a maximum in the $20 \mathrm{~nm}$ thick layered sample in the range of $550-600 \mathrm{~nm}$, in addition to the usual $\mathrm{BHJ}$ spectra. The inset in the figure shows the external quantum efficiency (EQE) of the fabricated devices by employing these thin copper phthalocyanines as a buffer layer where an increase in EQE efficiency of $75.94 \%$ was observed in the $\sim 10 \mathrm{~nm}$ thin layer device. However, a substantial decrease in quantum efficiency in the $\sim 20 \mathrm{~nm}$ deposited buffer layer was observed (51.96\%), which could be ascribed to increased resistance and charge recombination. It is remarkable to observe that in addition to poor efficiency, the EQE peaks shifted to the left, a bathochromic shift of $\sim 80 \mathrm{~nm}$ which could be ascribed to heavy charge recombination, increase in resistance and blocking of solar spectra by the thick films. Fig. 1b shows a $J-V$ characteristic response of the corresponding buffer layers in the solar cell devices. It can be conjectured from the obtained
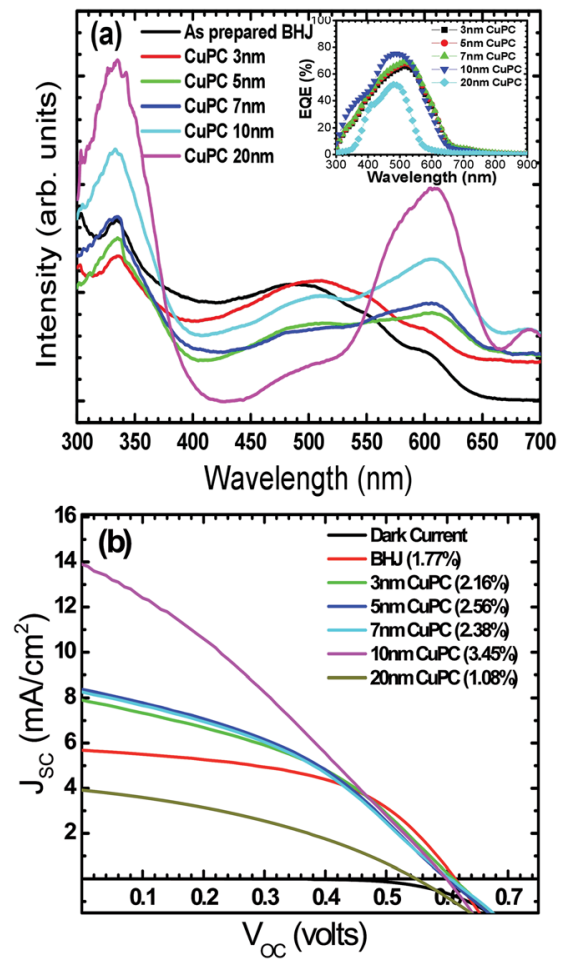

Fig. 1 (a) UV-visible absorption spectra of copper phthalocyanine buffer layers in respect of their thickness; inset shows EQE analysis. (b) Shows J-V characteristics of corresponding bulk-heterojunction solar cells. results that the efficiency obtained was maximum when a $\sim 10 \mathrm{~nm}$ thin film was grown on the glass/ITO/PEDOT:PSS layer, with the best performance of $3.45 \%$ with a $J_{\mathrm{SC}}$ value of $13.90 \mathrm{~mA} \mathrm{~cm} \mathrm{~cm}^{-2}$ and $V_{\mathrm{OC}}$ value of 0.60 volts, compared with the usual BHJ solar cell efficiency of $1.78 \%\left(J_{\mathrm{SC}}\right.$ of $5.76 \mathrm{~mA} \mathrm{~cm}^{-2}$ and $V_{\text {OC }}$ of 0.61 volts). It could be speculated that the lower mobility and short life time of the generated excitons, especially holes, limits the percolation path, owing to the slow mobility of the holes in comparison to electrons, leading to recombination. However, by the stacking of such a p-type buffer layer (CuPC) towards the anode, the excited photons are collected superbly by both the electrodes with efficient charge separation. Furthermore, it has been reported that the diffusion lengths of excitons in organic solar cells are up to $\sim 10 \mathrm{~nm}$, and in the present case, the best performance was found to be with the $\sim 10 \mathrm{~nm}$ thin layer which is very consistent with the reported results. It can be seen from Fig. 1b that the insertion of $\sim 3 \mathrm{~nm}$, $\sim 7 \mathrm{~nm}$ and $\sim 5 \mathrm{~nm}$ thin buffer films also shows an enhanced efficiency. However, the solar cell device with the $\sim 20 \mathrm{~nm}$ thin buffer layer (CuPC) shows a drastic low in efficiency of $1.08 \%$ with a $J_{\mathrm{SC}}$ of $3.83 \mathrm{~mA} \mathrm{~cm}^{-2}$ and $V_{\mathrm{OC}}$ of 0.54 volts with a lower fill factor. An effective increase in $J_{\mathrm{SC}}$ was noticed with an increase in the CuPC layer thickness up to $\sim 10 \mathrm{~nm}$, but the substantial decrease with a thickness of $\sim 20 \mathrm{~nm}$ reflects a reduced cell performance owing to an increase in the series resistance that hampers charge carrier transport. Additionally, augmented recombination between holes and electrons occurs while they are passing throughout the thick layer and such a thick buffer layer could decrease the number of photons arriving at the P3HT:PCBM blend due to an overlap in the absorption spectra of CuPC with P3HT:PCBM, as observed in the UV-vis results. These pioneering studies draw our curiosity to elucidate the influence of other metallophthalocyanines as thin buffer layers on the performance of BHJ solar cells by growing VoPC, NiPC, SnPC, TiPC, FePC, and InPC in the thicknesses of $\sim 5 \mathrm{~nm}$ and $\sim 10 \mathrm{~nm}$ that have been showing better performances.

Fig. 2 shows the current density versus voltage $(J-V)$ characteristics of fabricated devices with a thin interpolation of metallophthalocyanines (VoPC, NiPC, SnPC, TiPC, FePC, InPC)

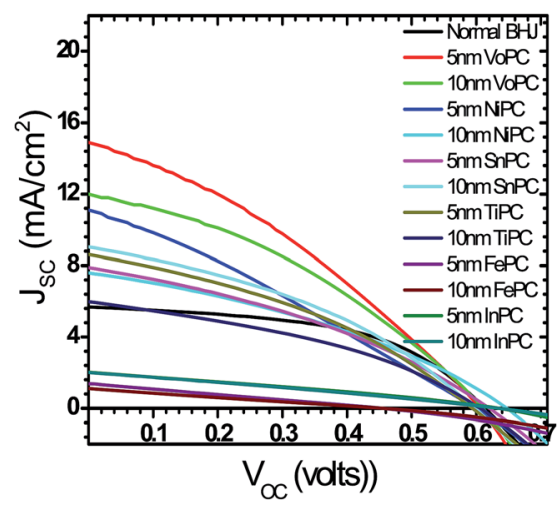

Fig. 2 Shows J-V characteristics of different metallophthalocyanines employed in bulk-heterojunction solar cells with various stacking layer thicknesses. 
under AM 1.5G conditions from the calibrated solar simulator with irradiation of $100 \mathrm{~mW} \mathrm{~cm}{ }^{-2}$. It has been observed that the bulk-heterojunction device performance in each transition metallophthalocyanine is different, even though the same thickness ( $\sim 5 \mathrm{~nm}, \sim 10 \mathrm{~nm})$ of buffer layers was inserted, which clearly reflects the sensitivity of the devices, with a slight alteration in any parameter heavily influencing the final performance. The usual $\mathrm{BHJ}$ device produces an efficiency of $1.78 \%$ ( $J_{\mathrm{SC}}$ of $5.78 \mathrm{~mA} \mathrm{~cm}{ }^{-2}, V_{\mathrm{OC}}$ of 0.6 volts and FF of 43 ), whereas the transition metal phthalocyanine with thickness of $\sim 5 \mathrm{~nm}$ and $\sim 10 \mathrm{~nm}$ shows an enhanced efficiency with a maximum $J_{\mathrm{SC}}$ value of $14.80 \mathrm{~mA} \mathrm{~cm}^{-2}, V_{\mathrm{OC}}$ of 0.60 , and $\mathrm{FF}$ of 53 with an overall conversion efficiency of $3.61 \%$ in the case of $\sim 5 \mathrm{~nm}$ of inserted VoPC, while $\sim 5 \mathrm{~nm}$ of NiPC shows a $J_{\mathrm{SC}}$ value of $11.15 \mathrm{~mA} \mathrm{~cm} \mathrm{~cm}^{-2}$ and the $\sim 10 \mathrm{~nm}$ NiPC shows a $J_{\mathrm{SC}}$ value of $8.98 \mathrm{~mA} \mathrm{~cm}{ }^{-2}$. However, the $V_{\mathrm{OC}}$ value is unaffected and remains the same with overall efficiencies of $3.13 \%$ and $2.52 \%$, respectively. It can be also found that for the sample with $\sim 5 \mathrm{~nm}$ of SnPC inserted, the $J_{\text {SC }}$ value decreases to $7.79 \mathrm{~mA} \mathrm{~cm}^{-2}$ with a lower fill factor of 38 with an efficiency of $2.14 \%$, but with $\sim 10 \mathrm{~nm}$ of SnPC there is a further lowering of the $J_{\mathrm{SC}}$ value to $7.49 \mathrm{~mA} \mathrm{~cm}{ }^{-2}$ with an efficiency of $2.11 \%$. The increase in the efficiency was obviously due to the enhancement in $J_{\mathrm{SC}}$, resulting from enhanced light harnessing, fast charge separation and hole migration and low recombination. It is further presumed that the possible enhanced efficiency in the $\sim 5 \mathrm{~nm}$ VoPC inserted buffer layer is owing to an enlargement in the built-in electric field between the anode and the active layer resulting in better charge transport. The device structure and energy diagram for the best device are presented in Fig. 3a and b, showing a proposed interface energy setup by using $\sim 5 \mathrm{~nm}$ of VoPC between PEDOT:PSS and the active layer. Additionally, a representative device structure and energy diagram for TiPC are shown in Fig. S2 (ESI $\dagger$ ) and it is worth noting that, in the case of $\sim 5 \mathrm{~nm}$ and $\sim 10 \mathrm{~nm}$ of TiPC, FePC and InPC, substantially lower efficiencies were noticed. The lowering in efficiency could be ascribed solely to the structure based on the phthalocyanines as no other parameters in the device were altered and the arrangement of the porphyrin ring of phthalocyanines and oxygen arranged as downhill/uphill over the PEDOT:PSS layer result in an enhanced recombination. In addition, the non- planer arrangement (TiPC, FePC, InPC) and the properties of the central metal also have a substantial influence which might affect the efficiency. But in the case of other metallophthalocyanines the main reasons for the enhancement in the efficiencies could be speculated to be based on the planar geometry of the porphyrin chain which can be flatly adhered to the PEDOT:PSS without being uphill/downhill, so the carriers are generated at the interface of P3HT:PCBM in the photoactive layer, which could form a tunnelling junction to increase the built-in electric field, an interfacial dipole layer to change the work function of electrodes or to protect the photoactive layer from damage. Metallophthalocyanines are introduced into $\mathrm{BHJ}$ solar cells in order to align the energy level and for easy collection of the charge carrier, as well as to extend the spectrum absorption range. This is in addition to their organic semiconductive nature which means they possess good chemical, heat, and light stability. ${ }^{31}$ Therefore, the increased efficiencies are attributed to the enhanced and efficient carrier (hole) transport from the photoactive layer to the PEDOT:PSS via the buffer layer which could effectively prevent the recombination of the charge carriers at the active layer/ITO interface because of the energy alignment of the highest occupied molecular orbital (HOMO) energy of the metal phthalocyanines with P3HT and because the generated holes can be extracted from the active layer to the anode and may easily form better percolating pathways maximizing high charge collection. The $V_{\mathrm{OC}}$ value showed no significant change in all the photovoltaic devices except at the FePC buffer layer application. According to the literature, it has been stated that the $V_{\mathrm{OC}}$ of polymer $\mathrm{BHJ}$ photovoltaic devices depends mainly on the relative energy levels of the donor and acceptor materials. The theoretical maximum $V_{\mathrm{OC}}$ is defined as the energy difference between the HOMO of the BHJ donor and the lowest unoccupied molecular orbital (LUMO) of the acceptor material; ${ }^{32}$ however, at this point we do not have clarity as to why it is lower in the FePC, but one speculative answer could be interfacial roughness and imperfections in the internal charge distribution of the film, as seen in the SEM images. Also, as reported in the literature, the electronic structure of its LUMO is quite different and is distributed in the $\mathrm{d}_{z}$ orbital. Kim et al. reported that inserting a $10 \mathrm{~nm}$ pentacene layer augmented the $V_{\mathrm{OC}}$ up to $0.73 \mathrm{~V}^{33}$

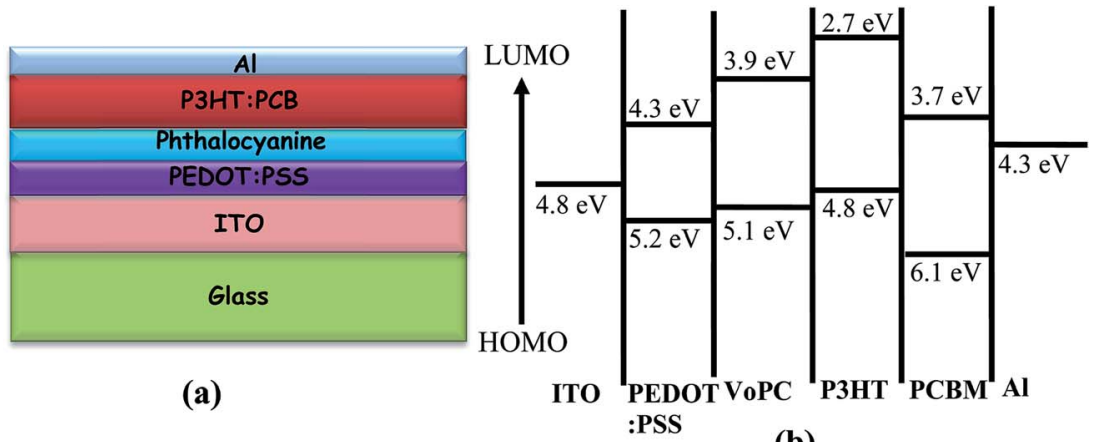

(b)

Fig. 3 (a) Depicts different layers of cell stacking. (b) Band diagram of representative metallophthalocyanines (VoPC) employed in bulk-heterojunction solar cells. 
Simultaneously it has also been influenced by the contacts between the active layer and the electrodes, the morphology of the active layer, insertion of a thin buffer layer and an interfacial dipole layer to shift the work functions of the $V_{\mathrm{OC}}$ of the electrodes.

The external quantum efficiencies (EQE) of the fabricated solar cell devices employing these metallophthalocyanines (VoPC, NiPC, SnPC, TiPC, FePC, InPC) as thin buffer layers are shown in Fig. 4. The spectra clearly reflect the change observed with a significant increase in quantum efficiency when $\sim 5 \mathrm{~nm}$ of VoPC, $\sim 10 \mathrm{~nm}$ of NiPC, and $10 \mathrm{~nm}$ of SnPC were applied, showing remarkably higher values than normal $\mathrm{BHJ}$ solar cells with quantum efficiencies of $69 \%, 70 \%$, and $67 \%$, respectively. However, in the case of the $\sim 5 \mathrm{~nm}$ and $\sim 10 \mathrm{~nm}$ films of TiPC, FePC and InPC, the quantum efficiencies were reduced to $43 \%$, $40 \%, 27 \%, 23 \%, 30 \%$, and $20 \%$, respectively, even lower than the usual BHJ. It is worth noting that by employing such metallophthalocyanines as thin buffer layers, the PCBM peak at $350 \mathrm{~nm}$ appears like a shoulder in almost all the fabricated cases, which might reflect a better intermixing and the reduction of boundary barriers when it was stacked between the PEDOT:PSS and the active layer. With NiPC, SnPC and VoPC the main peaks move towards the right of $520 \mathrm{~nm}$ from the normal $\mathrm{BHJ}(510 \mathrm{~nm})$ but in the case of NiPC, TiPC and InPC the main peaks do not move much from the normal BHJ. Furthermore, with TiPC a total shift in the overall peak towards the left of $\sim 78 \mathrm{~nm}$ is observed, which could be due to the high bandgap of titanium phthalocyanine $(2.37 \mathrm{eV})$ compared to the others. However, in the case of FePC the quantum efficiency peak is comparatively lower than for all the other metallophthalocyanines since the band gap of FePC is reported to be $1.82 \mathrm{eV}$. In all our external quantum efficiency results, there was a broad hump peak centered at $\sim 510 \mathrm{~nm}$ with narrow peaks at $\sim 690 \mathrm{~nm}$ in all the solar cells, showing that the device also harvests a small amount of photons at this position, which is consistent with the main peak of the UV-vis results. The higher EQE intensity in VoPC and NiPC could result from better charge collection efficiency, consistent with higher TOF mobility or a high charge generation yield and the application of thin metallophthalocyanines as a buffer layer for a better hole rectifier.

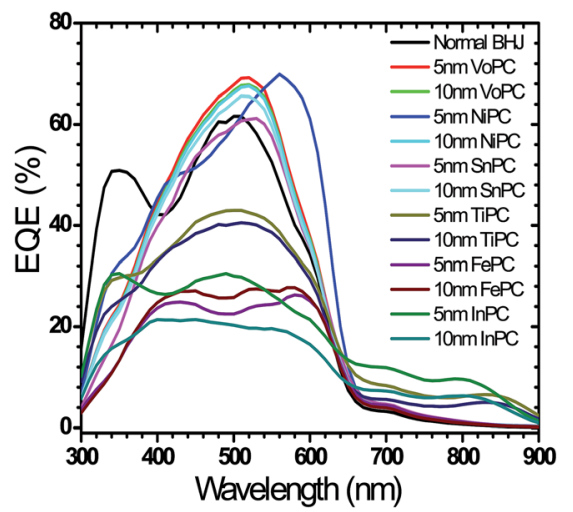

Fig. 4 Shows EQE of different metallophthalocyanines employed in bulk heterojunction solar cells with various stacking layer thicknesses.
Fig. 5a shows UV-vis spectra of the fabricated active layer with the stacking of glass/ITO/PEDOT:PSS/MoPC/P3HT:PCBM as an example (VoPC, NiPC, SnPC, TiPC, FePC) of grown $\sim 5 \mathrm{~nm}$ and $\sim 10 \mathrm{~nm}$ thin films where substantial solar band absorptions in the range of $\sim 600$ to $\sim 900 \mathrm{~nm}$ were observed. This clearly shows an extension of solar energy harnessing by employing metallophthalocyanines which might be helping to increase the $J_{\mathrm{SC}}$ value, impacting on the overall efficiency. It is predicted that since P3HT based $\mathrm{BHJ}$ has a band gap of $1.9 \mathrm{eV}$ that leads to solar cell harnessing up to a wavelength of $\sim 652 \mathrm{~nm}$, if the solar energy harnessing is augmented by employing new materials this might result in enhanced solar cell efficiencies, since the $J_{\mathrm{SC}}$ value is directly related to the optical absorption of the active layer. The fabricated active layer clearly shows a better and strong light harnessing ability of band intensities, particularly in the $\sim 10 \mathrm{~nm}$ thin layer stacking, although in the $\sim 5 \mathrm{~nm}$ buffer layer band intensities were also nice but weaker than above. However, only FePC shows a decreased absorbance, although it shows the strong P3HT absorbance. The rest of the metallophthalocyanines show the longer wavelength feature in addition to the regular 450$640 \mathrm{~nm}$ which is ascribed to the P3HT chains showing better spectral vibrionic bands. ${ }^{34}$ This remains unaltered for all the devices fabricated, suggesting that the stacking of the metallophthalocyanines $(\sim 5 \mathrm{~nm}, \sim 10 \mathrm{~nm})$ as a buffer layer may not affect the active layer absorbance. Additionally, the absorbance bands show an extra absorbance near the infrared in the 600-
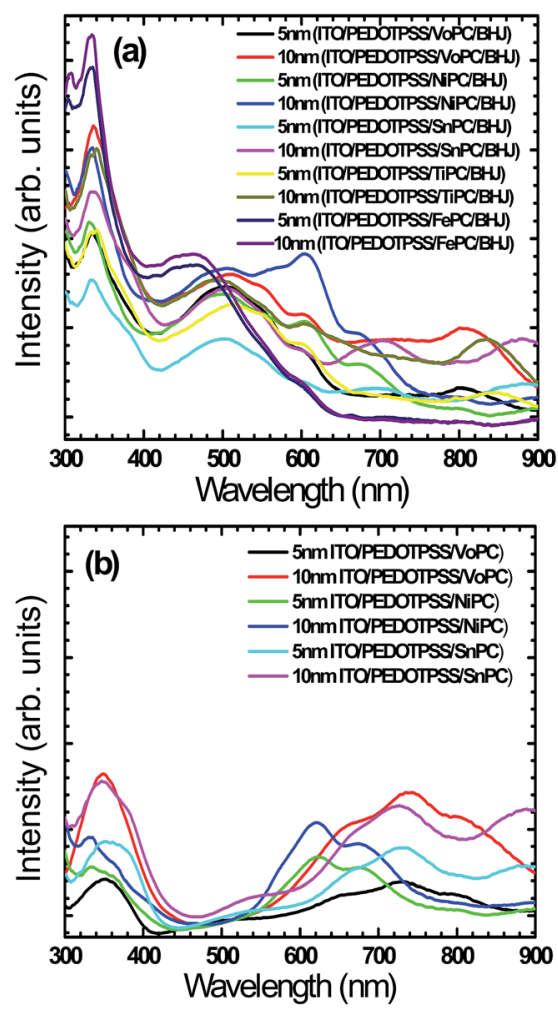

Fig. 5 (a) UV-visible absorption spectra of metallophthalocyanine buffer layers in respect of their thickness in BHJs. (b) Representative pristine UV-vis spectra of ITO/PEDOT:PSS/MOPC. 
$900 \mathrm{~nm}$ range arising from the interband $\pi-\pi^{*}$ transitions. It is worth noting that the solar cells where metallophthalocyanines were incorporated between the active and PEDOT:PSS layers show bathochromatic shifts with an enhanced $\pi-\pi *$ transition, indicating the strong interaction of the P3HT chain with phthalocyanine bands, and improved local structure ordering and better defined vibrionic side bands. The intensity and shape of the absorption features at $330 \mathrm{~nm}$ owing to the HOMO-LUMO transitions of PCBM remain the same and are undistinguishable in all the fabricated solar cell devices. This consistency is congruent with the literature where PCBM absorption shows little change after annealing. ${ }^{35}$ The impacts of the hierarchical placement of metal phthalocyanines suggest to us that there are no adverse impacts over the normal BHJs. Fig. 5b shows a representative UV-vis absorption of pristine glass/ITO/PEDOTPSS/phthalocyanines of VoPC, NiPC, SnPC in order to assess the impact of bare phthalocyanine insertion without the imposition of any active layer (P3HT:PCBM) where a substantial solar band absorption in the range of $\sim 600$ to $\sim 900 \mathrm{~nm}$ was clearly observed.
Fig. 6a-f shows the side view cross sectional scanning electron microscopy (SEM) of the as-prepared samples with a p-type buffer layer of VoPC, NiPC, SnPC and FePC, TiPC and InPC where the average film thickness of the active layer was reported to be $\sim 240 \mathrm{~nm}$. A clear demarcation of the active layer, ITO layer and dark gap of PEDOT:PSS and metallophthalocyanines is observed. It can be also visually observed that in general the active layer is smooth and homogenous and not much segregation of P3HT and PCBM particles is reported. However, in the case of FePC phthalocyanine comparatively rougher films were observed, but in the case of TiPC and InPC smoother films were also observed. In order to elucidate the device efficiency with stacked interfaces of $\sim 5 \mathrm{~nm}$ of VoPC or $\sim 10 \mathrm{~nm}$ of NiPC phthalocyanines, the buffer layer was flipped between the photoactive (P3HT:PCBM) and the vapour deposited Al cathodes, as shown in Fig. S1a and b. $\dagger$ It can be clearly observed from the figure that a substantially lower photocurrent $\left(J_{\mathrm{SC}}\right)$ of $0.46,0.54 \mathrm{~mA} \mathrm{~cm}^{-2}$, and $V_{\mathrm{OC}}$ of $0.61,0.55$ volts were observed, respectively, with an overall conversion efficiency of $\eta=0.029 \%$ and $0.048 \%$, as depicted in Fig. S1b. $\dagger$ The extraordinarily
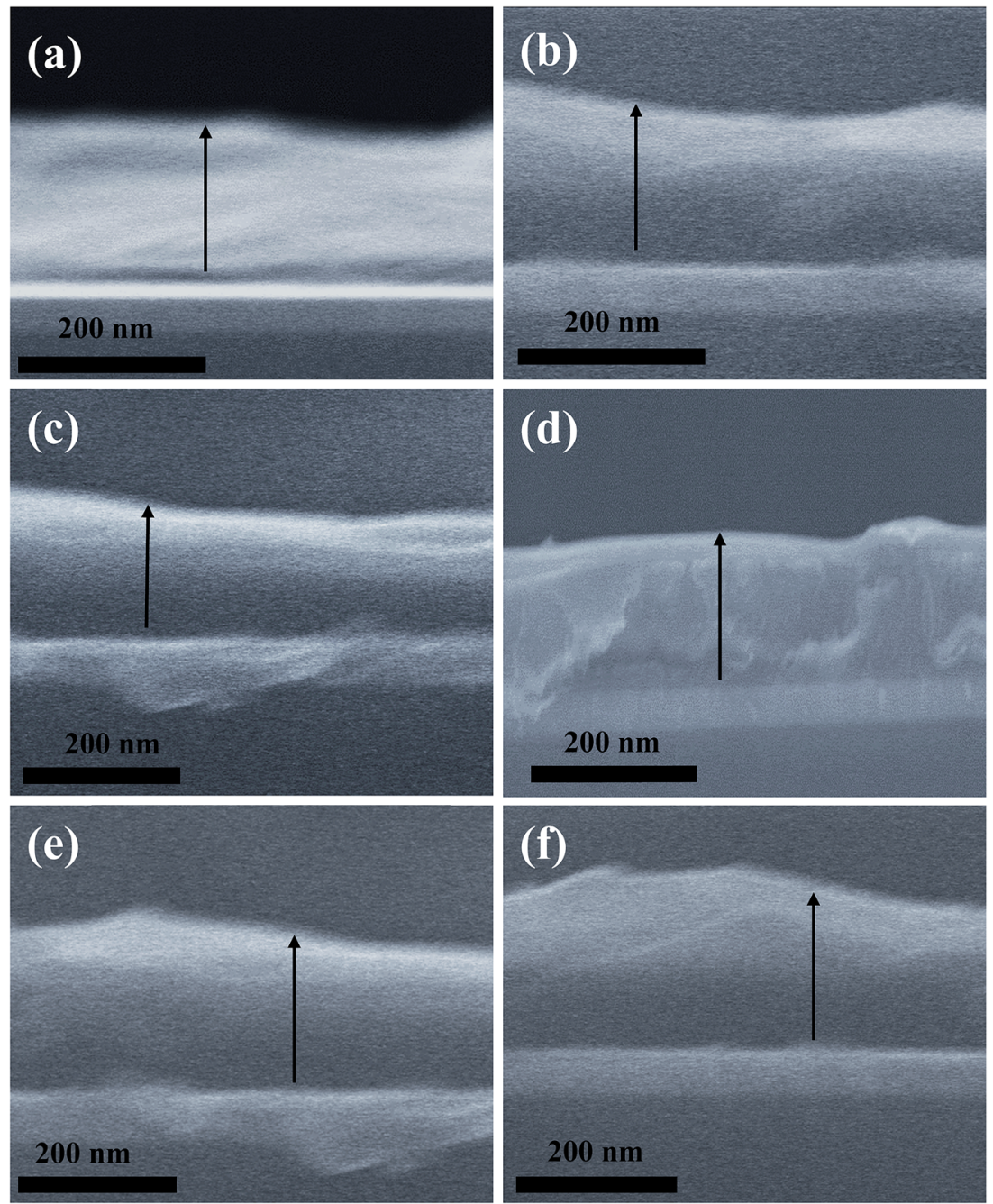

Fig. 6 Scanning electron microscopy (SEM) image of bulk heterojunction solar cell with metallophthalocyanines (a) $5 \mathrm{~nm}$ VoPC, (b) $10 \mathrm{~nm}$ NiPC, (c) $5 \mathrm{~nm} \mathrm{SnPC}$, (d) $5 \mathrm{~nm} \mathrm{FePC,} \mathrm{(e)} 5 \mathrm{~nm}$ TiPC and (f) $5 \mathrm{~nm} \operatorname{lnPC}$. 
reduced efficiency might be due to the possibility of a lower work function which is in close proximity with $\mathrm{Al}(4.2 \mathrm{eV})$, leading to lower electric field generation and poor charge collection at the cathode, since metallophthalocyanines are hole transporters and being on the electron transport side a heavily reduced charge tunnel junction transport and recombination will take place. Hence the movement of carriers must be severely hampered, resulting in enhanced thermalization. The external quantum efficiencies of the corresponding devices are shown in the inset of Fig. S1a. $\dagger$ They possess a substantially lower quantum efficiency of $15 \%$ and $28 \%$, respectively, with $\sim 5 \mathrm{~nm}$ of VoPC and $\sim 10 \mathrm{~nm}$ of NiPC inserted in the buffer layer. The UV-vis spectra of ITO/PEDOTP:SS/ P3HT:PCBM/VoPC and NiPC are shown in Fig. S1a, $\uparrow$ where a solar band absorption in the range of the P3HT chain main peak at $610 \mathrm{~nm}$ with transition metal phthalocyanine bands of $\sim 600$ to $\sim 750 \mathrm{~nm}$ was observed with improved vibrionic side bands and a reduction in complementary absorption spectra. The intensity and shape of absorption features at $330 \mathrm{~nm}$ owing to HOMO-LUMO transitions of PCBM remain the same and are undistinguishable in all the fabricated solar cells. It is also shown that a clear extension in solar energy harnessing by employing metallophthalocyanines has also been observed.

\section{Discussion}

Since the evaporation technique is well suited to thin multihierarchical structure photovoltaics with a sharp interface between the different layers in order to maximize the characteristics of each thin layer, deliberating on such a positive note, we have inserted thin p-type buffer layer metallophthalocyanines to obtain better photo-conversion and hole rectification, leading to improved efficiencies in $\mathrm{BHJ}$ devices. It is expected that a suitable buffer layer at the anode side that possesses an extended absorption, high transparency and holding p-type properties will be synergistically helpful in transferring holes efficiently towards the anodes. Since the electron mobility in PCBM $\left(2.0 \times 10^{-7} \mathrm{~m}^{2} \mathrm{~V}^{-1} \mathrm{~S}^{-1}\right)$ is about 4000 times higher than hole mobility $\left(5.0 \times 10^{-11} \mathrm{~m}^{2} \mathrm{~V}^{-1} \mathrm{~S}^{-1}\right),{ }^{36}$ use of a buffer may provide an advantage if their lower exciton binding energy and the distance that a charge carrier travels in the device (given by $d=\mu \tau F$ where $\mu$ is carrier mobility, $\tau$ is life time of charge carrier, $F$ is internal electric field) are optimized. The higher electron mobility causes an imbalance with the limited holes, which might cause enhanced recombinations, so an appropriate thin p-type buffer layer is needed to support the enhanced hole transfer to rectify this, but it should also not block solar light resulting in better percolation pathways. The metallophthalocyanines possess key parameters such as high transparency, and suitable band energy alignment and an efficient hole transporting ability and if they can extend the light harvesting ability along with a flatly sharp film interface, they will have a profound positive effect on the $J_{\mathrm{SC}}$ value and will be of great benefit for enhancement of BHJ solar cell efficiency. Owing to their characteristics they are quite appropriate with absorption spectra covering almost all the near visible region whereas the P3HT covers just $640 \mathrm{~nm}$; the formation of smooth films with a thermally stable nature, which also act as a barrier for the reduction of the hygroscopic nature of the PEDOT:PSS layer; and better hole transportation leading to an overall maximum solar cell efficiency of $3.61 \%$. However, except in VoPC, NiPC, SnPC and CuPC, we do not observe a positive effect on the devices, even though all metallophthalocyanines are categorised as p-type materials which can be conjectured to be the result of several factors, such as the central metal atom, planar and nonplanar complex orientation, and oxygen to metal bonding generating uphill and downhill tendencies of the central metal atom, as reported by Strohmaier et al. in studying phthalocyanines by STM. ${ }^{37}$ This also affects the optoelectronic properties, since the isoindole rings are similar in size in the planar forms (CuPC, VoPC, NiPC) with profoundly orbital mediated tunnelling by $\pi-\pi *$ orbitals on the aromatic ring on conjugation significantly affecting the conductivity and hence the inherent overall buffer layer property. However, probably in the non-planar metallophthalocyanines (TiPC, FePC, InPC) the structure orientation of oxygen downhill results in an enhanced distance between the PEDOT:PSS layer and the aromatic ring which reduces the tunnelling probability and overall resonance capability in congruence with the PEDOT:PSS layer. Moreover, the specific metallophthalocyanines and PCBM may also form a bilayer heterojunction cell because of good intermixing of the active (P3HT:PCBM) layer itself, giving some additional positive effects. The metallophthalocyanines acting as donor could be connected to the PCMB interface as an acceptor and excitons could be dissociated at the MOPC/PCBM interface, resulting in enhanced percolation. The vapour deposited phthalocyanine films are shining and smooth with good homogeneous distribution and a flat surface in a photovoltaic device is very useful to decrease current leakage and contact resistance. ${ }^{38}$ So, they can efficiently extract the holes and there is good band structure matching with metallophthalocyanines between the work function of the anode and the highest occupied molecular orbital of the phthalocyanines. The insertion of MOPC improves the device performance, especially the $J_{\mathrm{SC}}$ value, and expands the absorption spectra range. It has been also reported that the Al metal can effectively transfer the electron to the conjugated polymer with the sulfide species, and this feature makes it a potential cathode for polymeric electronics. ${ }^{39}$ Another study ${ }^{40}$ has also reported that $\mathrm{Cu}$ can react with $\mathrm{P} 3 \mathrm{HT}$ and form sulfide like species. These sulfide like species can improve the solar cell performance. It is believed that the formation of the P3HT-Al complexes will also play the same role in our case. With the help of P3HT-Al complexes and the Al-O-C bonds, there is better contact between the electrode and the active layer, resulting in an overall improved solar cell efficiency.

\section{Conclusions}

We have inserted a vapor deposited metallophthalocyanine (VoPC, NiPC, SnPC, TiPC, FePC, InPC) thin p-type buffer layer into BHJ solar cell devices with a cell structure of glass/ITO/ PEDOT:PSS/MoPC/P3HT:PCBM/Al in order to improve solar cell efficiencies. It has been observed that metallophthalocyanines of $\sim 5 \mathrm{~nm}$ and $\sim 10 \mathrm{~nm}$ thickness show an 
enhanced $J_{\mathrm{SC}}$ value (VoPC, NiPC, SnPC) with a maximum $J_{\mathrm{SC}}$ value of $14.80 \mathrm{~mA} \mathrm{~cm}{ }^{-2}, V_{\mathrm{OC}}$ of 0.60 , and $\mathrm{FF}$ of 51 with a conversion efficiency of $3.61 \%$ in the case of $\sim 5 \mathrm{~nm}$ of VoPC, while $\sim 5 \mathrm{~nm}$ of NiPC also shows a high $J_{\mathrm{SC}}$ value of $11.15 \mathrm{~mA}$ $\mathrm{cm}^{-2}$ and $\sim 10 \mathrm{~nm}$ of NiPC shows a $J_{\mathrm{SC}}$ value of $8.98 \mathrm{~mA} \mathrm{~cm}^{-2}$ with conversion efficiencies of $3.13 \%$ and $2.52 \%$, respectively. But some metallocyanines (TiPC, FePC, InPC) show a lower efficiency than normal BHJ cells. The enhanced efficiency in VoPC and NiPC might result from extended absorption, high transparency and holding p-type properties to transfer holes efficiently towards the anodes. Moreover, a better bicontinuous interpenetrating network is formed, which greatly reduces the exciton loss and improves the charge transport capability.

\section{Acknowledgements}

This work was carried out as a program supported by New Energy and Industrial Technology Development Organization (NEDO) of the Ministry of Economy, Trade, and Industry, Japan. Dr Khan thanks Dongguk University for selecting him for a faculty position.

\section{Notes and references}

1 C. J. Brabec, N. S. Sariciftci and J. C. Hummelen, Adv. Funct. Mater., 2001, 11, 15-26.

2 G. Yu, J. Gao, J. C. Hummelen, F. Wudl and A. J. Heeger, Science, 1995, 270, 1789-1791.

3 S. J. Lou, J. M. Szarko, T. Xu, L. P. Yu, T. J. Marks and L. X. Chen, J. Am. Chem. Soc., 2011, 133, 20661-20663.

4 M. Reyes-Reyes, K. Kim and D. L. Carroll, Appl. Phys. Lett., 2005, 87, 083506-083513.

5 J. Peet, J. Y. Kim, N. E. Coates, W. L. Ma, D. Moses, A. J. Heeger and G. C. Bazan, Nat. Mater., 2007, 6, 497-500.

6 J. K. Lee, W. L. Ma, C. J. Brabec, J. Yuen, J. S. Moon, J. Y. Kim, K. Lee, G. C. Bazan and A. J. Heeger, J. Am. Chem. Soc., 2008, 130, 3619-3623.

7 W. L. Ma, C. Y. Yang, X. Gong, K. Lee and A. J. Heeger, Adv. Funct. Mater., 2005, 15, 1617-1622.

8 K. I. Bolotin, K. J. Sikes, Z. Jiang, M. Kilma, G. Fudenberg, J. Hone, P. Kim and H. L. Stormer, Solid State Commun., 2008, 146, 351-355.

9 A. A. Balandin, S. Ghosh, W. Bao, I. Calizo, D. Teweldebrhan, F. Miao and C. N. Lau, Nano Lett., 2008, 8, 902-907.

10 T. M. Clarke and J. R. Durrant, Chem. Rev., 2010, 110, 67366767.

11 C. J. Brabec, G. Zerza, G. Cerullo, S. D. Silvestri, S. Luzzati, J. C. Hummelen and S. Sariciftci, Chem. Phys. Lett., 2001, 340, 232-236.

12 V. D. Mihailetchi, J. Wildeman and P. W. M. Blom, Phys. Rev. Lett., 2005, 94, 126602.

13 V. D. Mihailetchi, H. Xie, B. D. Boer, L. J. A. Koster and P. W. M. Blom, Adv. Funct. Mater., 2006, 16, 699-708.

14 J. Huang, G. Li and Y. Yang, Appl. Phys. Lett., 2005, 87, 112105.

15 T. J. Savenije, J. E. Kroeze, X. Yang and J. Loos, Adv. Funct. Mater., 2005, 15, 1260-1266.
16 P. Wurfel, Physics of Solar Cells, Wiley-VCH, Berlin, Germany, February 2009.

17 J. Huang, P. F. Miller, J. S. Wilson, A. J. de Mello, J. C. de Mello and D. D. C. Bradley, Adv. Funct. Mater., 2005, 15, 290-296.

18 C. Ionescu-Zanetti, A. Mechler, S. A. Carter and R. Lal, Adv. Mater., 2004, 16, 385-389.

19 C. J. Ko, Y. K. Lin, F. C. Chen and C. W. Chu, Appl. Phys. Lett., 2007, 90, 63509.

20 J. Ouyang, C. W. Chu, F. C. Chen, Q. Xu and Y. Yang, Adv. Funct. Mater., 2005, 15, 203-208.

21 G. de la Torre, C. G. Claessens and T. Torres, Chem. Commun., 2007, 2000-2015.

22 F. D'Souza and O. Ito, Chem. Commun., 2009, 4913-4928.

23 M. V. Martinez-Diaz, G. de la Torre and T. Torres, Chem. Commun., 2010, 46, 7090-7108.

24 G. Bottari, G. de la Torre, D. M. Guldi and T. Torres, Chem. Rev., 2010, 110, 6768-6816.

25 G. Bottari, J. A. Suanzes, O. Trukhina and T. Torres, J. Phys. Chem. Lett., 2011, 2, 905-913.

26 K. W. Hipps, X. Lu, X. D. Wang and U. Mazur, J. Phys. Chem., 1996, 100, 11207-11210.

27 X. Lu and K. W. Hipps, J. Phys. Chem. B, 1997, 101, 53915396.

28 N. Tao, Phys. Rev. Lett., 1996, 76, 4066-4069.

29 X. Lu, K. W. Hipps, X. D. Wang and U. Mazur, J. Am. Chem. Soc., 1996, 118, 7197-7202.

30 C. Zhang, S. W. Tong, C. Jiang, E. T. Kang, D. S. H. Chan and C. Zhu, Appl. Phys. Lett., 2008, 92, 083310-083313.

31 J. H. Zagal, S. Griveau, J. F. Silva, T. Nyokong and F. Bedioui, Coord. Chem. Rev., 2010, 254, 2755-2791.

32 C. J. Brabec, A. Cravino, D. Meissner, N. S. Sariciftci, T. Fromherz, M. T. Rispens, L. Sanchez and J. C. Hummelen, Adv. Funct. Mater., 2001, 11, 374-380.

33 J. H. Kim, S. Y. Huh, T. Kim and H. Lee, Appl. Phys. Lett., 2008, 93, 143305-143313.

34 Y. Kim, S. Cook, S. M. Tuladhar, S. A. Choulis, J. Nelson, J. R. Durrant, D. D. C. Bradley, M. Giles, I. McCulloch, C. Ha and M. Ree, Nat. Mater., 2006, 5, 197-203.

35 D. Chirvase, J. Parisi, J. C. Hummelen and V. Dyakonov, Nanotechnology, 2004, 15, 1317-1323.

36 V. D. Mihailetchi, J. K. J. van Duren, P. W. M. Blom, J. C. Hummelen, R. A. J. Janssen, J. M. Kroon, M. T. Rispens, W. J. H. Verhees and M. M. Wienk, Adv. Funct. Mater., 2003, 13, 43-46.

37 R. Strohmaier, C. Ludwig, J. Petersen, B. Gompf and W. Eisenmenger, J. Vac. Sci. Technol., B, 1996, 14, 1079-1082. 38 M. G. Varnamkhasti, H. R. Fallah, M. Mostajaboddavati, R. Ghasemi and A. Hassanzadeh, Sol. Energy Mater. Sol. Cells, 2012, 98, 379-384.

39 Q. D. Ling, S. Li, E. T. Kang, K. G. Neoh, B. Liu and W. Huang, Appl. Surf. Sci., 2002, 199, 74-82.

40 B. Reeja-Jayan and A. Manthiram, Sol. Energy Mater. Sol. Cells, 2010, 94, 907-914. 